\section{Prevalência de disfunção sexual em primigestas, no Município de Rio Branco, Acre, Brasil}

\author{
Prevalence of sexual dysfunction in primigravidae \\ in Rio Branco, Acre State, Brazil \\ Prevalencia de disfunción sexual en primigestas en
el municipio de Río Branco, Acre, Brasil
}

Adriani Castro de Lima 1

Leila Maria Geromel Dotto 1

Marli Villela Mamede 2

\footnotetext{
1 Programa de Pós-graduação em Saúde Coletiva

Universidade Federal do Acre, Rio Branco, Brasil.

2 Escola de Enfermagem de Ribeirão Preto, Universidade de São Paulo, Ribeirão Preto, Brasil.

Correspondência A. C. Lima

Programa de Pós-graduação em Saúde Coletiva, Universidade Federal do Acre. Campus Universitário, BR-364, km 04 - Distrito Industrial C, Rio Branco, AC 69920-900, Brasil. adriani_80@yahoo.com.br
}

\section{Abstract}

This study aimed to estimate the prevalence of sexual dysfunction prior to and during the first pregnancy. This was a cross-sectional study using postpartum interviews with 778 primigravidae who were married or in stable unions and had given birth at the two maternity hospitals in Rio Branco, Acre State, Brazil, from February $1^{\text {st }}$ to July 31 st, 2010. Median age was 20 years, 45\% were adolescents, $19 \%$ had completed elementary school, 30\% had paid jobs, and 86.5\% had used public healthcare services (SUS) for childbirth. Prevalence of sexual dysfunction was $23.9 \%$ prior to pregnancy and $67.7 \%$ during pregnancy. Decreased libido was present in $20.2 \%$ prior to pregnancy and $51 \%$ during pregnancy. Decreased vaginal lubrication occurred in $29.1 \%$ during pregnancy. Dyspareunia was present in $1.2 \%$ prior to pregnancy and $14.4 \%$ during pregnancy. Some 3.3\% reported sexual dissatisfaction prior to pregnancy, as compared to $10.8 \%$ during pregnancy. Women with more schooling showed higher prevalence of sexual dysfunction during (as compared to before) their first pregnancy.

Physiological Sexual Dysfunction; Sexual Health; Pregnancy; Women

\section{Resumo}

O objetivo deste estudo foi estimar a prevalência das disfunções sexuais antes e durante a gravidez por meio de um estudo transversal, realizado com 778 primigestas, com companheiro, que pariram nas duas maternidades de Rio Branco, Acre, Brasil, entre 1o de fevereiro e 31 de julho de 2010, utilizando entrevista no pós-parto. A mediana de idade das primigestas foi de 20 anos, $45 \%$ eram adolescentes, $19 \%$ possuíam escolaridade até o ensino fundamental; $30 \%$ tinham trabalho remunerado, 86,5\% utilizaram o serviço público (SUS) para os partos. A prevalência de disfunção sexual foi de 23,9\% antes da gravidez e de 67,7\% na gravidez. Antes da gestação, a falta de desejo sexual foi de 20,2\% e, na gestação, de 51\%. A diminuição da lubrificação vaginal durante a gestação foi de 29,1\%, a dispareunia foi de 1,2\% antes da gestação e de 14,4\% na gestação, 3,3\% tiveram insatisfação sexual antes da gravidez, e 10,8\%, na gestação. Os achados indicam que as mulheres estudadas apresentaram maior prevalência de disfunção sexual durante a primeira gravidez do que no período anterior a essa gestação.

Disfunção Sexual Fisiológica; Saúde Sexual; Gravidez; Mulheres 


\section{Introdução}

A vida sexual, durante a gravidez, é afetada por inúmeros fatores decorrentes das mudanças físicas, emocionais próprias da gestação e também pelas crenças sobre sexualidade adquiridas antes da gestação, transmitidas seja pela cultura ou pela religião. A maneira como a mulher vivencia a sexualidade, a receptividade dela e do parceiro para com a gravidez, a concepção sobre o significado de se tornarem pais e mães, a identidade e o papel sexual feminino e fatores econômicos são aspectos que influenciam fortemente a sexualidade durante essa fase 1,2 .

O tema sexualidade tem despertado atenção, nos últimos tempos, em várias áreas do conhecimento e tem sido tratado sob diferentes enfoques; no entanto, estudos que o relacionam ao ciclo gravídico-puerperal são raros, pois se trata de assunto que ainda causa desconforto, tanto para a gestante quanto para os profissionais que a atendem.

A gravidez é, sem dúvida, um acontecimento especial na vida de cada mulher, e a primeira gestação é uma transição marcante, semelhante à menarca e à menopausa que representam uma passagem de um estágio para outro no desenvolvimento feminino, com fortes implicações na sexualidade, na autoimagem e nos relacionamentos interpessoais. O processo de transformação da mulher em mãe traz consigo adaptações e preocupações de diferentes naturezas, quer seja com o seu corpo, com o parto, com a chegada do bebê e especialmente com sua vida sexual que pode estar prejudicada $3,4,5$.

A presença de algum transtorno na sexualidade feminina, seja de interrupção ou de alteração de qualquer uma das fases da resposta sexual (desejo, excitação, orgasmo e resolução), pode acarretar o surgimento de disfunções sexuais $6,7,8,9$. Essas podem se manifestar por falta, excesso ou desconforto e/ou dor associada ao ato sexual de maneira persistente ou recorrente 10.

A resposta sexual saudável é definida como um conjunto de quatro etapas: desejo, excitação, orgasmo e resolução 11,12, mas estudos revelam que as mulheres não apresentam a resposta sexual de maneira fixa, inerte, na mesma ordem exposta. Ao contrário, elas descrevem a resposta sexual normal com uma sequência flexível, variável, ou seja, as fases da resposta sexual feminina podem se sobrepor umas às outras, sofrendo combinações de respostas psicológicas e físicas 6,7 .

Há relatos de que $40 \%$ das gestantes apresentaram diminuição na frequência sexual, no primeiro trimestre gestacional, quando comparado ao período pré-gravídico. No segundo trimestre, a diminuição foi de $30 \%$, e, no terceiro trimestre, foi maior que $60 \% 13$.

Parece existir, durante o período de gravidez, uma clara diminuição do interesse sexual assim como um decréscimo da atividade sexual 14 . Gökyildiz \& Beji 1 identificaram que 21,3\% das grávidas descreveram ter medo de manter relações sexuais no terceiro trimestre.

Fatores emocionais relacionados à gestação que podem ser vivenciados tanto pela gestante como pelo parceiro também alteram a sexualidade feminina; entre tais fatores, estão o ajustamento aos novos papéis sociais, a qualidade do relacionamento do casal, alterações de humor, aceitação do sexo nesse período, entre outros 13 . A dor durante a penetração, a diminuição na lubrificação vaginal e no desejo também contribuem para a disfunção sexual 15 .

É fato que a função sexual adequada do indivíduo é um determinante importante de satisfação e qualidade de vida, no entanto, estudos demonstram uma alta prevalência de disfunção sexual, sendo reconhecida, pela Organização Mundial da Saúde (OMS), como um problema de saúde pública que necessita ser melhor estudado 16 .

Este estudo tem como objetivo avaliar a função sexual de primigestas, no Município de Rio Branco, Acre, Brasil, procurando caracterizar o perfil socioeconômico das primigestas e estimar a prevalência das disfunções sexuais (dispareunia, ausência de desejo, insatisfação e diminuição da lubrificação vaginal) antes e durante a gravidez.

\section{Material e métodos}

A pesquisa caracterizou-se como estudo de corte transversal, realizado a partir de um estudo matriz denominado Saúde Reprodutiva de Primigestas: Análise de Fatores Relacionados ao Tipo de Parto, realizado em Rio Branco.

As gestantes elegíveis para o estudo foram as mulheres que pariram nas duas maternidades existentes no município, uma pública, que atende somente ao Sistema Único de Saúde (SUS), e outra no Hospital Filantrópico, que atende ao SUS, a convênios e a particulares que tinham domicílio no Município de Rio Branco e que estavam dando à luz a seu primeiro filho.

Para o cálculo da amostra, foi considerado, como base, o número de partos ocorridos no ano de 2007, em Rio Branco, que foi de 7.094 partos, dos quais 2.644 ocorreram em primigestas, correspondendo a uma média de aproximadamente 220 partos mensais em primigestas (dados da Coordenação Materno-Infantil, Secretaria de 
Estado da Saúde do Acre; 2010). Adotando-se uma confiabilidade de $95 \%$, um poder de $80 \%$ e uma razão de chances estimada de 2,0 para os fatores de exposição analisados, foi determinado que seriam necessárias em torno de 804 mulheres para a amostra, sendo a coleta de dados definida por um período de seis meses que ocorreu de primeiro de fevereiro a 31 de julho de 2010, nas duas maternidades do município, totalizando 887 parturientes entrevistadas (com perdas e recusas de $10,95 \%)$.

$\mathrm{Na}$ amostra total de primigestas do estudo matriz (887), encontram-se 107 mulheres que não tiveram parceiro durante a gravidez, e duas delas foram restringidas de ter relações sexuais por seus médicos; portanto, fizeram parte deste estudo 778 mulheres que tiveram companheiro durante o período gestacional. Todas as participantes assinaram o termo de consentimento livre e esclarecido.

A coleta de dados foi realizada por meio de entrevista, na unidade de alojamento conjunto, em torno de 12 horas de pós-parto, como forma de respeitar as primeiras horas de recuperação do pós-parto imediato. $\mathrm{O}$ instrumento utilizado foi elaborado com vistas a alcançar os objetivos propostos pelo estudo, sendo constituído de duas partes. A primeira parte tratava de informações sobre dados sociodemográficos, a gestação e foi adaptada do estudo Capital Social e Fatores Psicossociais Associados à Prematuridade e ao Baixo Peso ao Nascer, coordenado por Maria do Carmo Leal (Escola Nacional de Saúde Pública Sergio Arouca/Fiocruz). A segunda parte tratava de questões relacionadas à saúde sexual cujo conteúdo foi construído com base no estudo de Gökyildiz \& Beji ${ }^{1}$ que analisou os efeitos da gravidez sobre a vida sexual de mulheres grávidas. Foram, então, utilizadas 13 questões, de múltipla escolha, sobre saúde sexual do Pregnancy and Sexual Function Questionnaire (PSFQ) daquele estudo, sendo que, para oito questões, foram solicitadas respostas para antes da gestação, para o primeiro, o segundo e o terceiro trimestre de gravidez (de acordo com o instrumento adotado). O instrumento PSFQ, utilizado por Gökyildiz \& Beji ${ }^{1}$, foi validado para a língua portuguesa por Amaral 17.

O instrumento de coleta de dados foi submetido a um teste-piloto, com 50 puérperas, com a finalidade de avaliar a compreensão das perguntas, tendo, então, sido efetuadas as adequações semânticas, e, assim, estabelecida a sua versão final. Essas mulheres não fizeram parte do estudo.

Para operacionalizar a coleta de dados, foram treinadas cinco auxiliares de pesquisa (acadêmicas dos cursos de Enfermagem e de Saúde Coletiva da Universidade Federal do Acre - UFAC). O treinamento constituiu-se de uma parte teórica (6 horas) e de uma parte prática (8 horas), o treinamento prático foi realizado durante o testepiloto.

As variáveis utilizadas neste estudo foram:

- Sociodemográficas: idade, cor da pele, situação conjugal, escolaridade, ocupação, renda familiar (em salários-mínimos), tipo de serviço (atendimento pelo SUS e plano de saúde/particular) e maternidade;

- Disfunção sexual: a disfunção sexual (binária com valor sim e não) foi considerada como variável resposta (variável dependente), a qual pode ser apresentada em qualquer trimestre da gestação ou mesmo ao longo do período gestacional. Nesta pesquisa, foi considerada, como disfunção sexual, a presença de um ou mais dos seguintes domínios da resposta sexual feminina: desejo sexual, lubrificação vaginal, dor na relação sexual e satisfação sexual.

Para a definição operacional da variável disfunção sexual e sua classificação, foram consideradas as respostas aos itens relacionados à saúde sexual do instrumento de coleta de dados que tratam especificamente de cada domínio descrito:

- Desejo sexual: pergunta "Com que frequência você sentia desejo sexual?”, a resposta considerada foi "não sentia" e pergunta "Quem tinha iniciativa para a relação sexual?", a resposta considerada foi "sempre meu parceiro", demonstrando assim a ausência do desejo ou do interesse sexual;

- Lubrificação: pergunta "Quanto a sua lubrificação vaginal durante a relação sexual de vocês, mudou alguma coisa durante a gravidez?", sendo considerada a resposta "sim, diminuiu", demonstrando a diminuição de excitação sexual;

- Dor: pergunta "Você tem dor durante a relação sexual?", sendo considerada a resposta "sim, sempre”, demonstrando a presença de dor sexual no ato sexual;

- Satisfação: pergunta "Qual é a sua satisfação com relação à relação sexual com seu parceiro?", sendo considerada a resposta "extremamente insatisfeita", demonstrando insatisfação sexual.

Os dados coletados foram analisados com o apoio do programa estatístico SPSS, versão 13.0 (SPSS Inc., Chicago, Estados Unidos). Para a análise das disfunções sexuais antes da gravidez e durante a gestação, foi utilizado o teste de homogeneidade de Wilcoxon, considerando-se estatisticamente significativos os valores de $\mathrm{p}<0,05$.

$\mathrm{O}$ estudo matriz teve seu projeto de pesquisa submetido ao Comitê de Ética em Pesquisa (CEP) da UFAC e foi aprovado sob o protocolo no 23107.005912/2009-21, recebendo autorização das duas instituições onde a coleta de dados foi realizada. 


\section{Resultados}

A amostra de primigestas estudadas é representada por $45,1 \%$ de mulheres com até 19 anos de idade, ou seja, adolescentes, sendo que a idade das entrevistadas variou de 13 a 43 anos (mediana: 20, média: 21,52 e DP: 5,24 ), 22,6\% conseguiram chegar ao nível superior, independente de completá-lo; $48 \%$ referiram renda familiar mensal menor que dois salários-mínimos, e a maioria $(86,5 \%)$ dos partos teve o SUS como fonte pagadora (Tabela 1).

Quanto aos fatores indicativos de disfunção sexual, no período pré-gravídico e nos trimestres gestacionais, ocorreu um declínio no desejo sexual feminino na gravidez quando comparado a antes da gravidez, como também entre os períodos de gestação. Quando perguntado sobre quem tinha a iniciativa para a relação sexual, observa-se que o estrato "sempre o parceiro" teve um aumento conforme a evolução da gestação, bem como o número de mulheres que não aceitavam a inovação do parceiro na relação sexual (Tabela 2).

A frequência de mulheres que estavam extremamente insatisfeitas com a relação sexual também cresceu, conforme a gravidez evoluía, chegando a $10,5 \%$ no terceiro trimestre; e a dor, durante a atividade sexual, relatada pelas primigestas no terceiro trimestre teve uma frequência de $47,9 \%$.

Mudanças no padrão da lubrificação vaginal foram identificadas na gravidez pela maioria das mulheres, sendo que a diminuição ocorreu em $24,3 \%$ no primeiro trimestre, no segundo trimestre, em 25,9\% e, no terceiro, em $27,8 \%$ das gestantes.

Com relação às variáveis: frequência do desejo sexual, iniciativa para a relação sexual, aceitação de inovação na relação sexual, satisfação com a relação sexual, dor durante a relação sexual e lubrificação vaginal, todas as diferenças encontradas entre os trimestres da gestação apresentaram significância estatística (Tabela 2).

A prevalência de algum tipo de disfunção sexual, antes da gravidez, foi bem menor do que a apresentada durante a primeira gravidez. Quando analisada por trimestre gestacional, observou-se um acréscimo da disfunção sexual conforme o aumento da idade gestacional em que a prevalência da ocorrência de qualquer um dos problemas na função sexual (falta de desejo, diminuição da lubrificação, insatisfação e presença de dor durante as relações sexuais) passa de $54,1 \%$ no primeiro trimestre para $66,3 \%$ no terceiro trimestre da gestação (Tabela 3 ).

Nos quatro domínios indicativos de disfunção sexual aqui estudados, observou-se, nos trimestres gestacionais, um crescimento na fre quência de todos, conforme o avanço da gestação. Nota-se claramente que a "falta de desejo sexual" foi o problema sexual que teve maior prevalência dentre os demais, tanto antes como durante a gravidez, por essas primigestas.

A Tabela 4 apresenta a associação da disfunção sexual antes e durante a gravidez com algumas variáveis selecionadas (sociodemográficas e relacionadas à gravidez e vida sexual da primigesta durante a gestação).

Ter até 18 anos (RP: 1,642; IC95\%: 1,039-2,596), ser estudante (RP: 1,770; IC95\%: 1,109-2,826) ou dona de casa (RP: 1,652; IC95\%: 1,088-2,509) e ter estudado até o ensino médio ou mais (RP: 1,430; IC95\%: 1,028-1,991) aumentou a chance de ter disfunção sexual antes da gravidez. Após serem ajustadas por idade, nenhuma variável apresentou significância estatística.

A presença de disfunção sexual durante a gravidez esteve associada ao planejamento da gravidez (RP: 1,433; IC95\%: 1,058-1,940), permanecendo com significância estatística após ser ajustada por idade (RP: 1,448; IC95\%: 1,0591,979); as demais varáveis, depois de serem ajustadas por idade, não apresentaram significância estatística.

\section{Discussão}

Nesta pesquisa, destaca-se que quase metade das primigestas tinha até 19 anos, representando o início da maternidade na fase da adolescência, resultado que se aproxima de outros estudos 18 . Esses dados corroboram com a afirmativa de que a gravidez na adolescência ainda é um dado alarmante, e esse fenômeno ocorre mundialmente; sendo que sete países, entre eles o Brasil, respondem por metade de todos os partos de adolescentes no mundo 19.

Nesse período da vida, a adolescente passa por conflitos familiares, maturação sexual, formação e consolidação de valores e comportamentos que irão reger sua vida adulta, além da cobrança de maior responsabilidade por seus atos e uma postura de definição na área profissional. Nesse contexto, o acontecimento de uma gravidez pode gerar sentimentos contraditórios de prazer, de culpa e de baixa autoestima, podendo interferir na sua função sexual, dando início a problemas ou ressaltando-os 20,21.

A gravidez quando ocorre em meninas até os 19 anos, sendo considerada uma situação de risco, pode ser vista como um problema quando não é desejada/planejada, especialmente nas classes socioeconomicamente desfavorecidas. Esse segmento populacional apresenta maior 
Distribuição das variáveis socioeconômicas e planejamento da gravidez das primigestas atendidas nas maternidades de Rio Branco, Acre, Brasil, 2010.

\begin{tabular}{|c|c|c|}
\hline \multirow[t]{2}{*}{ Variáveis } & \multicolumn{2}{|c|}{ Total } \\
\hline & $n$ * & $\%$ \\
\hline \multicolumn{3}{|l|}{ Idade (anos) } \\
\hline$\leq 19$ & 351 & 45,1 \\
\hline$\geq 20$ & 427 & 54,9 \\
\hline \multicolumn{3}{|l|}{ Cor autodeclarada } \\
\hline Branca & 151 & 19,4 \\
\hline Não branca & 627 & 80,6 \\
\hline \multicolumn{3}{|l|}{ Mora com companheiro } \\
\hline Sim & 668 & 85,9 \\
\hline Não & 110 & 14,1 \\
\hline \multicolumn{3}{|l|}{ Escolaridade } \\
\hline Até Ensino Médio incompleto & 353 & 45,4 \\
\hline Ensino Médio completo ou mais & 425 & 54,6 \\
\hline \multicolumn{3}{|l|}{ Ocupação } \\
\hline Estudante & 190 & 24,7 \\
\hline Dona de casa & 345 & 44,9 \\
\hline Ocupações diversas & 233 & 30,3 \\
\hline \multicolumn{3}{|l|}{ Renda familiar (salários-mínimos) ** } \\
\hline$>2$ & 358 & 48,0 \\
\hline $2-5$ & 292 & 39,1 \\
\hline 5 ou mais & 96 & 12,9 \\
\hline \multicolumn{3}{|l|}{ Tipo de atendimento no parto } \\
\hline SUS & 673 & 86,5 \\
\hline Particular/convênio & 105 & 13,5 \\
\hline \multicolumn{3}{|l|}{ Maternidade } \\
\hline I & 262 & 33,7 \\
\hline II & 516 & 66,3 \\
\hline \multicolumn{3}{|l|}{ Gravidez planejada } \\
\hline Não & 430 & 55,6 \\
\hline Sim & 343 & 44.4 \\
\hline
\end{tabular}

* Diferenças de valores se devem aos missings (não resposta);

** Salário-mínimo: $\mathrm{R} \$ 510,00$.

exposição às DST e à AIDS, ao uso de drogas, a acidentes e às diferentes formas de violência 20 .

Frente aos achados deste estudo, recomendase um olhar mais ampliado para os adolescentes, incorporando programas de educação sexual nas escolas, de maneira a possibilitar a informação sobre os problemas e as dificuldades de uma gravidez precoce, realizando parcerias dos governos com empresas privadas e estatais na busca de inserção dos jovens no mercado de trabalho, melhorando a renda e também o desempenho escolar. No entanto, um aspecto essencial é a capacitação de profissionais de saúde que atuam no pré-natal para que consigam fazer uma abor- dagem segura e eficaz sobre o funcionamento sexual nessa fase da vida.

No Brasil, em 2005, segundo o Departamento de Informática do SUS (DATASUS; http://www2. datasus.gov.br/DATASUS/index.php?area $=0205$, acessado em 10/Mai/2012), do total de nascidos vivos, $21,8 \%$ eram de mães adolescentes (até 19 anos) em 2010, esse número correspondeu a 19,3\%, sendo a Região Norte responsável por $15 \%$ desses partos, e Rio Branco responsável por $0,28 \% 25$. Quando se compara o número de partos ocorridos em adolescentes nos anos de 2005 e 2010, observa-se que ainda é muito alto, motivo de grande preocupação devido aos resultados 
Distribuição dos fatores indicativos de disfunção sexual no período pré-gravídico e nos trimestres gestacionais, em primigestas atendidas nas maternidades de Rio Branco, Acre, Brasil, 2010.

\begin{tabular}{|c|c|c|c|c|c|c|c|c|c|c|c|c|}
\hline \multirow[t]{3}{*}{ Fatores indicativos de disfunção sexual } & \multirow{2}{*}{\multicolumn{2}{|c|}{$\begin{array}{l}\text { Antes da } \\
\text { gravidez }\end{array}$}} & \multicolumn{6}{|c|}{ Trimestre gestacional } & \multicolumn{4}{|c|}{ Valor de $p$ * } \\
\hline & & & \multicolumn{2}{|c|}{1 으 } & \multicolumn{2}{|c|}{ 2o } & \multicolumn{2}{|c|}{ 3o } & \multirow{2}{*}{$\begin{array}{l}\text { Antes da } \\
\text { gravidez até } \\
\text { 1ㅇ trimestre }\end{array}$} & \multirow{2}{*}{$\begin{array}{c}\text { 1ㅇ- 2으 } \\
\text { trimestre }\end{array}$} & \multirow{2}{*}{$\begin{array}{l}\text { 1ㅇ- 3으 } \\
\text { trimestre }\end{array}$} & \multirow{2}{*}{$\begin{array}{l}\text { 2ㅇ- 3으 } \\
\text { trimestre }\end{array}$} \\
\hline & $\mathbf{n}$ & $\%$ & $\mathrm{n}$ & $\%$ & $\mathrm{n}$ & $\%$ & $\mathrm{n}$ & $\%$ & & & & \\
\hline \multicolumn{13}{|l|}{ Frequência do desejo sexual } \\
\hline Não sentia & 11 & 1,4 & 106 & 13,6 & 133 & 17,1 & 187 & 24,1 & & & & \\
\hline Uma vez ou mais ao dia & 253 & 32,6 & 180 & 23,2 & 139 & 17,9 & 93 & 12,0 & & & & \\
\hline 3 ou 4 vezes por semana & 364 & 46,8 & 207 & 26,6 & 164 & 21,1 & 109 & 14,0 & & & & \\
\hline Uma a duas vezes por semana & 137 & 17,6 & 217 & 27,9 & 244 & 31,4 & 231 & 29,7 & & & & \\
\hline Uma vez a cada duas semanas & 8 & 1,0 & 37 & 4,8 & 59 & 7,6 & 64 & 8,2 & & & & \\
\hline Menos que uma vez por mês & 4 & 0,5 & 30 & 3,9 & 38 & 4,9 & 93 & 12,0 & 0,004 & 0,002 & $<0,001$ & 0,016 \\
\hline \multicolumn{13}{|l|}{ Quem tinha iniciativa para a relação } \\
\hline \multicolumn{13}{|l|}{ sexual } \\
\hline Sempre eu & 13 & 1,7 & 28 & 3,8 & 33 & 4,5 & 36 & 5,4 & & & & \\
\hline Às vezes eu & 14 & 1,8 & 26 & 3,5 & 36 & 4,9 & 21 & 3,2 & & & & \\
\hline Os dois com a mesma frequência & 573 & 73,7 & 390 & 52,4 & 326 & 44,6 & 254 & 38,2 & & & & \\
\hline Às vezes meu parceiro & 25 & 3,2 & 82 & 11,0 & 91 & 12,4 & 80 & 12,0 & & & & \\
\hline Sempre meu parceiro & 153 & 19,7 & 218 & 29,3 & 245 & 33,5 & 274 & 41,2 & $<0,001$ & 0,002 & $<0,001$ & $<0,001$ \\
\hline \multirow{2}{*}{\multicolumn{13}{|c|}{$\begin{array}{l}\text { Aceita inovação do parceiro na relação } \\
\text { sexual }\end{array}$}} \\
\hline & & & & & & & & & & & & \\
\hline Geralmente aceita com prazer & 649 & 83,5 & 430 & 57,9 & 333 & 45,7 & 250 & 37,1 & & & & \\
\hline Aceita relutante & 79 & 10,2 & 149 & 20,1 & 144 & 19,8 & 119 & 17,7 & & & & \\
\hline Frequentemente recuso & 31 & 4,0 & 87 & 11,7 & 144 & 19,8 & 173 & 25,7 & & & & \\
\hline Geralmente recuso & 18 & 2,3 & 77 & 10,4 & 108 & 14,8 & 131 & 19,5 & $<0,001$ & $<0,001$ & $<0,001$ & $<0,001$ \\
\hline \multicolumn{13}{|l|}{ Está satisfeita com a relação sexual } \\
\hline Extremamente insatisfeita & 26 & 3,3 & 45 & 6,1 & 51 & 7,0 & 71 & 10,5 & & & & \\
\hline Moderadamente insatisfeita & 7 & 0,9 & 34 & 4,6 & 40 & 5,5 & 33 & 4,9 & & & & \\
\hline Pouco insatisfeita & 8 & 1,0 & 25 & 3,4 & 30 & 4,1 & 34 & 5,0 & & & & \\
\hline Pouco satisfeita & 23 & 3,0 & 82 & 11,1 & 114 & 15,6 & 148 & 21,9 & & & & \\
\hline Moderadamente satisfeita & 150 & 19,3 & 233 & 31,4 & 231 & 31,6 & 192 & 28,4 & & & & \\
\hline Extremamente satisfeita & 564 & 72,5 & 323 & 43,5 & 265 & 36,3 & 198 & 29,3 & $<0,001$ & $<0,001$ & $<0,001$ & $<0,001$ \\
\hline \multicolumn{13}{|l|}{ Tem dor durante a relação sexual } \\
\hline Às vezes & 83 & 10,7 & 164 & 22,0 & 206 & 28,1 & 230 & 33,8 & & & & \\
\hline Sempre & 9 & 1,2 & 59 & 7,9 & 74 & 10,1 & 96 & 14,1 & & & & \\
\hline Nunca & 686 & 88,2 & 523 & 70,1 & 453 & 61,4 & 354 & 51,5 & $<0,001$ & $<0,001$ & $<0,001$ & $<0,001$ \\
\hline \multicolumn{13}{|l|}{ Lubrificação vaginal mudou ** } \\
\hline Não & - & - & 372 & 50,0 & 352 & 48,2 & 313 & 45,1 & & & & \\
\hline Sim, diminuiu & - & - & 181 & 24,3 & 189 & 25,9 & 193 & 27,8 & & & & \\
\hline Sim, aumentou & - & - & 191 & 25,7 & 190 & 26,0 & 188 & 27,1 & & 0,037 & 0,004 & $<0,001$ \\
\hline
\end{tabular}

* Teste de homogeneidade de Wilcoxon;

** Nessa variável, não foi realizada a pergunta no período antes da gravidez.

negativos para a mãe e para o bebê que podem estar associados com a gravidez precoce ocorrida em adolescentes 22 .

Mesmo permanecendo sexualmente ativas, verificou-se, neste estudo, que o declínio da frequência e do desejo sexual foram problemas citados pelas primigestas, sendo que o terceiro tri- mestre gestacional foi o período em que se constatou maior ocorrência de disfunção sexual. A ausência do desejo sexual aumentou conforme a evolução dos trimestres gestacionais. Em outros estudos, também se observou a diminuição na frequência e no desejo sexual durante a gravidez, sendo que, no terceiro trimestre, a diminuição da 
Tabela 3

Prevalência da disfunção sexual e de cada domínio indicativo de disfunção sexual, antes e durante os trimestres da gravidez de primigestas atendidas nas maternidades de Rio Branco, Acre, Brasil, 2010.

\begin{tabular}{|c|c|c|c|c|c|c|c|c|c|c|}
\hline \multirow{3}{*}{$\begin{array}{l}\text { Domínios indicativos de disfunção } \\
\text { sexual }\end{array}$} & \multicolumn{2}{|c|}{ Antes da gravidez } & \multicolumn{6}{|c|}{ Trimestre gestacional } & \multicolumn{2}{|c|}{ Na gravidez } \\
\hline & \multirow[t]{2}{*}{$n$ * } & \multirow[t]{2}{*}{$\%$} & \multicolumn{2}{|c|}{ 1으 } & \multicolumn{2}{|c|}{ 2oㅇ } & \multicolumn{2}{|c|}{ 3으 } & \multirow[t]{2}{*}{$n$ * } & \multirow[t]{2}{*}{$\%$} \\
\hline & & & $n$ * & $\%$ & $n$ * & $\%$ & $n$ * & $\%$ & & \\
\hline Falta de desejo sexual & 157 & 20,2 & 273 & 35,5 & 309 & 39,7 & 365 & 46,9 & 393 & 51,0 \\
\hline Diminuição da lubrificação vaginal & - & - & 181 & 24,3 & 189 & 25,9 & 193 & 24,8 & 219 & 29,1 \\
\hline Dor durante a relação sexual & 9 & 1,2 & 59 & 7,9 & 74 & 10,1 & 96 & 14,1 & 109 & 14,4 \\
\hline Insatisfação sexual & 26 & 3,3 & 45 & 6,1 & 51 & 7,0 & 71 & 10,5 & 82 & 10,8 \\
\hline Disfunção sexual & 186 & 23,9 & 418 & 54,1 & 448 & 58,4 & 489 & 66,3 & 524 & 67,7 \\
\hline
\end{tabular}

* Diferenças de valores se devem aos missings (não resposta).

Tabela 4

Associação entre presença de disfunção sexual e variáveis sociodemográficas e fatores relacionados à gravidez, no período antes e durante a gravidez de primigestas atendidas nas maternidades de Rio Branco, Acre, Brasil, 2010.

\begin{tabular}{|c|c|c|c|}
\hline \multirow[t]{2}{*}{ Variáveis } & \multicolumn{3}{|c|}{ Disfunção sexual antes da gravidez } \\
\hline & RP & IC95\% & Valor de $p$ \\
\hline \multicolumn{4}{|l|}{ Idade (anos) } \\
\hline$\leq 18$ & 1,642 & $1,039-2,596$ & 0,034 \\
\hline $19-25$ & 1,067 & $0,675-1,686$ & 0,782 \\
\hline 26 ou mais & 1,000 & & \\
\hline Valor de $p$ de tendência & & & 0,037 \\
\hline \multicolumn{4}{|l|}{ Renda (salários-mínimos) } \\
\hline$<2$ & 1,290 & $0,753-2,209$ & 0,353 \\
\hline $2-5$ & 0,943 & $0,539-1,651$ & 0,838 \\
\hline 5 ou mais & 1,000 & & \\
\hline Valor de $\mathrm{p}$ de tendência & & & 0,144 \\
\hline \multicolumn{4}{|l|}{ Ocupação } \\
\hline Estudante & 1,770 & $1,109-2,826$ & 0,017 \\
\hline Dona de casa & 1,652 & $1,088-2,509$ & 0,019 \\
\hline Ocupações diversas & 1,000 & & \\
\hline Valor de p de tendência & & & 0,016 \\
\hline \multicolumn{4}{|l|}{ Escolaridade } \\
\hline Ensino Médio completo ou mais & 1.430 & $1,028-1,991$ & 0,034 \\
\hline Até Ensino Médio incompleto & 1,000 & & \\
\hline \multirow[t]{2}{*}{ Variáveis } & \multicolumn{3}{|c|}{ Disfunção sexual na gravidez } \\
\hline & RP & IC95\% & Valor de $p$ \\
\hline \multicolumn{4}{|l|}{ Idade (anos) } \\
\hline$\leq 18$ & 1,067 & $0,705-1,615$ & 0,758 \\
\hline $19-25$ & 0,935 & $0,630-1,388$ & 0,738 \\
\hline 26 ou mais & 1,000 & & \\
\hline Valor de $p$ de tendência & & & 0,797 \\
\hline
\end{tabular}




\begin{tabular}{|c|c|c|c|}
\hline \multirow[t]{2}{*}{ Variáveis } & \multicolumn{3}{|c|}{ Disfunção sexual na gravidez } \\
\hline & RP & $\mathrm{IC} 95 \%$ & Valor de $p$ \\
\hline \multicolumn{4}{|l|}{ Renda (salários mínimos) } \\
\hline$<2$ & 1,129 & $0,692-1,842$ & 0,627 \\
\hline $2-5$ & 0,822 & $0,501-1,347$ & 0,437 \\
\hline 5 ou mais & 1,000 & & \\
\hline Valor de $p$ de tendência & & & 0,240 \\
\hline \multicolumn{4}{|l|}{ Ocupação } \\
\hline Estudante & 1,304 & $0,864-1,969$ & 0,206 \\
\hline Dona de casa & 1,188 & $0,836-1,688$ & 0,337 \\
\hline Ocupações diversas & 1,000 & & \\
\hline Valor de $\mathrm{p}$ de tendência & & & 0,196 \\
\hline \multicolumn{4}{|l|}{ Escolaridade } \\
\hline Ensino Médio completo ou mais & 1,292 & $0,953-1,753$ & 0,099 \\
\hline Até Ensino Médio incompleto & 1,000 & & \\
\hline \multicolumn{4}{|l|}{ Gravidez planejada } \\
\hline Não & 1,433 & $1,058-1,940$ & 0,020 \\
\hline Sim & 1,000 & & \\
\hline \multicolumn{4}{|l|}{ Mora com companheiro } \\
\hline Não & 1,554 & $0,978-2,470$ & 0,062 \\
\hline Sim & 1,000 & & \\
\hline
\end{tabular}

IC95\%: intervalo de 95\% de confiança; RP: razão de prevalência ajustada por idade.

frequência das relações sexuais ocorreu de forma abrupta 1,13 .

Ao se compararem os resultados que foram obtidos nesta pesquisa com estudos realizados em outros países, verificou-se que, no Canadá, houve uma diminuição do desejo sexual no final da gestação em 58\% de 140 grávidas; em Portugal, $32,5 \%$ de 188 grávidas também tiveram diminuição no desejo sexual, e, na Turquia, a prevalência foi de $59 \%$ em 150 grávidas 1,22,23. A redução drástica no desejo sexual no terceiro trimestre também é congruente com outros achados como os da Turquia que destaca o terceiro trimestre como o período gestacional que teve maior ocorrência de redução 24 .

Quanto à tomada de iniciativa pela gestante para começar a relação sexual, observou-se, ao longo da gestação, um aumento desse comportamento quando comparado com o período pré-gravídico e os trimestres gestacionais entre si, encontrando-se um aumento à medida que a gravidez evoluiu. No entanto, outros estudos apontam uma diminuição da iniciativa para o início da relação sexual pelas primigestas no período pré-gravídico e gravídico, cenário contrário ao mostrado neste estudo 1 .

A satisfação da gestante quanto à relação sexual com o parceiro também foi outro domínio da função sexual que apresentou declínio ao longo dos trimestres, sendo que, do primeiro para o terceiro trimestre, existe uma diferença de quase $7 \%$ de insatisfação. Na pesquisa da Turquia, esse declínio se mostrou mais acentuado quando comparado aos resultados desta pesquisa, visto que houve uma diferença de $26 \%$ do primeiro para o terceiro trimestre 1 . Esses achados corroboram os resultados da presente pesquisa ao revelarem que as mulheres diminuem a satisfação sexual ao longo da gestação.

Estudo realizado em Fortaleza (Ceará), em 2001, com 4.753 médicos ginecologistas, mostrou que $83,7 \%$ das dificuldades sexuais apresentadas pelas pacientes nos consultórios médicos eram queixas encobertas por outras doenças, ou seja, os problemas sexuais eram abordados como queixa secundária, e que apenas $16,3 \%$ delas correspondiam à queixa principal 25 . A literatura mostra uma prevalência da disfunção sexual feminina, como queixa principal, variando de $16,3 \%$ a $74,2 \% 18,23,26,27,28$.

A prevalência de disfunção sexual feminina antes da gravidez, neste estudo, foi de $23,9 \%$, no entanto, apesar de esse valor não ser considerado tão alto como muitos trabalhos apontam, cabe ressaltar que, antes da gravidez, só foram avaliados três domínios da disfunção sexual, uma vez que a questão relacionada à diminuição da lubrificação vaginal não foi perguntada às primigestas quanto ao período antes da gravidez; além de considerar que a amostra foi 
composta por $45 \%$ de adolescentes, condição que pode fazer diferença em termos de experiência sexual.

A prevalência da dispareunia encontrada na literatura é de $13 \%$ em mulheres não grávidas e de $23,2 \%$ em gestantes 8,27 . Neste trabalho, a queixa de dor, durante as relações sexuais, foi crescente de um trimestre para o outro, atingindo um ápice $(48,5 \%)$ ao final da gestação, sendo os resultados semelhantes aos de alguns autores 1,28.

Problemas relacionados ao desejo sexua também são encontrados nos estudos com uma prevalência de $11 \%$ em mulheres não grávidas e de $25 \%$ de perda de desejo em gestantes 8,22 . Esse valor, mesmo sendo menor do que o encontrado neste trabalho, mostra que realmente há uma modificação no desejo sexual durante a gravidez, e que um número importante de gestantes são acometidas pela ausência de desejo.

Ribeiro et al. 28 encontraram problemas na lubrificação de 35\% das gestantes, resultado próximo ao desta pesquisa.

Nesta pesquisa, a prevalência da disfunção sexual foi menor antes da gestação e maior durante a gravidez. A prevalência da disfunção sexual durante a gestação, apresentada neste estudo, chama a atenção, principalmente, pela faixa etária em que estão vivendo as primigestas, ou seja, uma população muito jovem, muitas vezes, iniciando-se na vida sexual, em relacionamentos recentes, o que representa uma questão relevante para a saúde dessas mulheres.

O problema sexual mais prevalente foi a ausência do desejo sexual, seguido da diminuição da lubrificação. A ausência do desejo sexual, a insatisfação, a dispareunia e a diminuição na lubrificação vaginal aumentaram nas mulheres com a progressão da gestação. Muitas vezes, essas disfunções não são percebidas pelas mulheres como algo que esteja associado com a sua saúde ou, quando conseguem perceber, sentem vergonha e receio de expor o problema e, portanto, não procuram ajuda profissional durante o pré-natal. Torna, portanto, oportuno e salutar que os profissionais estejam preparados e sensibilizados para acolher e intervir nas questões relacionadas à função sexual na gestação cuja dimensão se faz tão presente na vida das mulheres e na gestação em especial.

Acresce-se que a sexualidade humana é um tema bastante desafiador para os profissionais de saúde, pois, além da dificuldade de abordagem do tema, há uma evidente escassez de instrumentos que auxiliem no diagnóstico preciso das disfunções sexuais, e, de uma forma geral, as queixas das mulheres são basicamente obtidas na clínica, sem uma sistematização, por meio do autorrelato das pacientes. Nesse sentido, instru- mentos autoaplicáveis e que contemplem todas as dimensões do funcionamento sexual podem tornar-se uma importante ferramenta na identificação de problemas sexuais e contribuir na captação dos acometidos com esses problemas.

Este estudo apresenta algumas limitações. Primeiro, diz respeito ao funcionamento sexual feminino, que, a priori, é um construto de difícil mensuração. A ausência de instrumentos ou de critérios fidedignos para a classificação dos problemas relacionados à resposta sexual feminina, como distúrbios do desejo, da excitação, do orgasmo, da dor, entre outros, é uma limitação enfrentada nos estudos sobre a resposta sexual feminina e, em especial, na gestação 29,30. Estudos prospectivos e longitudinais poderiam ajudar a compreender os efeitos da gravidez sobre a função sexual. Outra limitação relaciona-se ao instrumento selecionado para o estudo, o PSFQ, uma vez que as questões destinadas para avaliar o funcionamento sexual na gestação não incorporam todas as dimensões da resposta sexual feminina, especialmente aquelas relacionadas ao componente psicossocial, visto ser essa dimensão um importante marcador de disfunção sexual feminina. Acrescenta-se ainda a necessidade de pesquisas que venham contribuir para o refinamento do PSFQ, tanto do ponto de vista conceitual dos construtos relacionados ao funcionamento sexual na gestação como do estabelecimento de um sistema de pontuação para cada domínio estudado.

Existem outros instrumentos utilizados em estudos internacionais e que já foram validados para a população brasileira, mas não em gestantes, como o Female Sexual Function Inventory $31 \mathrm{e}$ o Female Sexual Function Index 16,32. Tais instrumentos têm se revelado como ferramentas importantes para a avaliação clínica de problemas sexuais femininos.

O Scale for Quality of Sexual Function (QSF), embora não específico para a gestação, é um outro instrumento que se mostra promissor para o estudo da função sexual, especialmente por permitir a sua utilização para ambos os gêneros, o que seria uma alternativa para o estudo do funcionamento sexual de casais 33 .

Reforça-se, assim, que a eleição pelo PSFQ, neste trabalho, ocorreu por ele ter sido desenvolvido especificamente para mulheres em gestação, por ele permitir identificar problemas relacionados à disfunção sexual nesse período de vida das mulheres e especialmente por ele ter sido validado para a língua portuguesa com grávidas do Município de Rio Branco 33 . 


\section{Resumen}

El objetivo de este estudio fue estimar la prevalencia de las disfunciones sexuales, antes y durante el embarazo. Es un estudio transversal realizado con 778 primigestas, con pareja, que dieron a luz en las dos maternidades de Río Branco, Acre, Brasil, entre el 1o de febrero y el 31 de julio de 2010, mediante entrevista en el posparto. La media de edad de las primigestas fue de 20 años, un $45 \%$ eran adolescentes, un $19 \%$ poseían escolaridad hasta la enseñanza fundamental; un 30\% tenían trabajo remunerado, un $86,5 \%$ utilizaron el servicio público (SUS) para los partos. La prevalencia de la disfunción sexual fue de un 23,9\% antes del embarazo y de un $67,7 \%$ durante el embarazo. Antes de la gestación, la falta de deseo sexual fue de un 20,2\%, y en la gestación de un 51\%. Disminución de la lubrificación vaginal durante la gestación fue de un 29,1\%, la dispareunia fue de un 1,2\% antes de la gestación y un 14,4\% durante la gestación, un 3,3\% sufrieron insatisfacción sexual antes del embarazo y un 10,8\%, en el embarazo. Los hallazgos indican que las mujeres estudiadas presentaron una mayor prevalencia de disfunción sexual durante el primer embarazo que en el período anterior a esta gestación.

Disfunción Sexual Fisiológica; Salud Sexual; Embarazo; Mujeres

\section{Colaboradores}

A. C. Lima participou da concepção, elaboração do projeto, coleta de dados, interpretação dos dados, redação do artigo e aprovação final da versão. L. M. G. Dotto participou da elaboração do projeto, coleta de dados, interpretação dos dados, redação do artigo e aprovação final da versão. M. V. Mamede contribuiu na interpretação dos dados, redação do artigo, revisão do conteúdo do texto e aprovação final da versão.

\section{Agradecimentos}

A realização desta investigação foi viabilizada por meio da colaboração acadêmica estabelecida entre o Programa de Pós-graduação em Saúde Coletiva da Universidade Federal do Acre e o Programa de Pós-graduação em Saúde Pública e Meio Ambiente da Fundação Oswaldo Cruz, a qual vem sendo apoiada com recursos do Conselho Nacional de Desenvolvimento Científico e Tecnológico (CNPq: Edital Casadinho UFAC-Fiocruz, processo no 620024/2008-9) e da Coordenação de Aperfeiçoamento de Pessoal de Nível Superior.

\section{Referências}

1. Gökyildiz S, Beji NK. The effects of pregnancy on sexual life. J Sex Marital Ther 2005; 31:201-15.

2. Salim NR, Araújo NM, Gualda DMR. Corpo e sexualidade: a experiência de um grupo de puérperas. Rev Latinoam Enferm 2010; 18:732-9.

3. Silveira CSP, Ferreir MMC. Autoconceito da grávida: fatores associados. Millenium - Revista do IPV 2011; (40):53-67.

4. Hernadez JAE. Gravidez do primeiro filho: papéis sexuais, ajustamento conjugal e emocional. Psicol Teor Pesqui 2008; 24:133-41.
5. Alves AM, Gonçalves CSF, Martins MA, Silva ST, Auweter TC, Zagonel IPS. A enfermagem e puérperas primigestas: desvendando o processo de transição ao papel materno. Cogitare Enferm 2007; 12:416-27.

6. Basson R, Leiblum S, Brotto L, Derogatis L, Fourcroy J, Fugl-Meyer K, et al. Revised definitions of women's sexual dysfunction. J Sex Med 2004; 1:40-8.

7. Basson R. Women's sexual dysfunction: revised and expanded definitions. CMAJ 2005; 172:1327-33. 
8. Ferreira ALCG, Souza AI, Amorim MMR. Prevalência das disfunções sexuais femininas em clínica de planejamento familiar de um hospital escola no Recife, Pernambuco. Rev Bras Saúde Matern Infant 2007; 7:143-50.

9. Antonioli RS, Simões D. Abordagem fisioterapêutica nas disfunções sexuais femininas. Rev Neurociênc 2010; 18:267-74.

10. Abdo CHN. Descobrimento sexual do Brasil: para curiosos e estudiosos. São Paulo: Summus; 2004.

11. American Psychiatric Association. Manual diagnóstico e estatístico de transtornos: DSM-IVTRTM. 4a Ed. Porto Alegre: Editora Artmed; 2002.

12. Abdo CHN, Fleury HJ. Revisão de literatura. Aspectos diagnósticos e terapêuticos das disfunções sexuais femininas. Rev Psiquiatr Clín (São Paulo) 2006; 33:162-7.

13. Savall ACR, Mendes AK, Cardoso FL. Perfil do comportamento sexual na gestação. Fisioter Mov 2008; 21:61-70.

14. Silva AI, Figueiredo B. Sexualidade na gravidez após o parto. Psiquiatria Clínica 2005; 25:253-64.

15. Rodrigues CEG. Dificuldades no retorno à atividade sexual nos primeiros seis meses após o parto [Dissertação de Mestrado]. Pelotas: Universidade Católica de Pelotas; 2009.

16. Thiel RRC, Dambros M, Palma PCR, Thiel M, Riccetto CLZ, Ramos MF. Tradução para português, adaptação cultural e validação do Female Sexual Function Index. Rev Bras Ginecol Obstet 2008; 30:504-10.

17. Amaral TLM. Validação do pregnancy and sexual function questionnaire sobre as mudanças na vida sexual durante a gravidez [Dissertação de Mestrado]. Rio Branco: Universidade Federal do Acre; 2012.

18. Leite APL, Campos AAS, Dias ARC, Amed AM, Souza E, Camano L. Prevalence of sexual dysfunction during pregnancy. Rev Assoc Med Bras 2009; 55:563-8.

19. World Health Organization. Adolescent pregnancy: a culturally complex issue. Bull World Health Organ 2009; 87:410-1.

20. Gurgel MGI, Alves MDS, Vieira NFC, Pinheiro PNC, Barroso GT. Gravidez na adolescência: tendência na produção científica de enfermagem. Esc Anna Nery Rev Enferm 2008; 12:800-6.
21. Gomes R, Fonseca EMGO, Veiga AJMO. A visão da pediatria acerca da gravidez na adolescência: um estudo bibliográfico. Rev Latinoam Enferm 2002; 10:408-14.

22. Naldoni LMV, Pazmiño MAV, Pezzan PAO, Pereira SB, Duarte G, Ferreira CHJ. Evaluation of sexual function in Brazilian pregnant women. J Sex Marital Ther 2011; 37:116-29.

23. Pauleta JR, Pereira NM, Graca LM. Sexuality during pregnancy. J Sex Med 2010; 7(1 Pt 1):136-42.

24. Bartellas E, Crane JM, Daley M, Bennett KA, Hutchens D. Sexuality and sexual activity in pregnancy. BJOG 2000; 107:964-8.

25. Aslam G, Aslam D, Kizilyar A, Ispahi Ç, Esen A. A prospective analysis of sexual functions during pregnancy. Int J Impot Res 2005; 17:154-7.

26. Abdo CHN, Oliveira Jr. WM. O ginecologista brasileiro frente às queixas sexuais femininas: um estudo preliminar. RBM Rev Bras Med 2002; 59:179-86.

27. Cerejo AC. Disfunção sexual feminina: prevalência e fatores relacionados. Revista Portuguesa de Clínica Geral 2006; 22:701-20.

28. Ribeiro MC, Nakamura MU, Abdo CHN, Torloni MR, Scanavino MT, Mattar R. Gravidez e diabetes gestacional: uma combinação prejudicial à função sexual feminina? Rev Bras Ginecol Obstet 2011; 33:219-24.

29. Costa VDM, Pinto MJC. Análise prospectiva da resposta sexual feminina na gestação. Revista Latinoamericana de Medicina Sexual 2012; 1:5-13.

30. Binik YM. The DSM diagnostic criteria for dyspareunia. Arch Sex Behav 2010; 39:292-303.

31. Graham CA. The DSM diagnostic criteria for female orgasmic disorder. Arch Sex Behav 2010; 39:256-70.

32. Hentschel H, Alberton DL, Capp E, Goldim JR, Passos EP. Validação do Female Sexual Function Index (FSFI) para uso na língua portuguesa. Revista do Hospital de Clínicas de Porto, 2007;27:10-4.

33. Pereira VM, Silva ACO, Nardi AE, Heinemann LAJ. Tradução e adaptação transcultural para o português brasileiro da Scale for Quality of Sexual Function (QSF). Rev Psiquiatr Rio Gd Sul 2011; 33:87-97.

Recebido em 13/Nov/2012

Versão final reapresentada em 01/Mar/2013

Aprovado em 13/Mar/2013 